\title{
Complications of UDT
}

Complications should be prevented by applying proper techniques and strictly following UDT rules, such as haematuria, oedema of the urinary bladder wall, bladder spasm, urethral trauma, autonomic dysreflexia attack have been reported, as well as bladder over-distension when the filling is continued too long.

The risk for symptomatic urinary tract infection (UTI) warrants antibiotic prophylaxis when UDT is done. 